\title{
FINITELY GENERATED $\delta$-SUPPLEMENTED MODULES ARE AMPLY $\delta$-SUPPLEMENTED
}

\section{RACHID TRIBAK}

(Received 6 November 2011)

\begin{abstract}
Let $R$ be a commutative ring. It is shown that if an $R$-module $M$ is a sum of $\delta$-local submodules and a semisimple projective submodule, then every finitely generated submodule of $M$ is $\delta$-supplemented. From this result, we conclude that finitely generated $\delta$-supplemented modules over commutative rings are amply $\delta$-supplemented.
\end{abstract}

2010 Mathematics subject classification: primary 16D10; secondary 16D60, 16D99.

Keywords and phrases: $\delta$-small submodule, $\delta$-local module, $m$ - $\delta$-local module, $\delta$-supplemented module, amply $\delta$-supplemented module.

\section{Introduction}

Throughout this paper $R$ will denote an associative commutative ring with identity and all modules are unital $R$-modules. Recall that a submodule $N$ of a module $M$ is said to be $\delta$-small in $M$, written $N \ll_{\delta} M$, provided $M \neq N+X$ for any proper submodule $X$ of $M$ with $M / X$ singular. Let $L$ be a submodule of a module $M$. A submodule $K$ of $M$ is called a $\delta$-supplement of $L$ in $M$ provided $M=L+K$ and $M \neq L+X$ for any proper submodule $X$ of $K$ with $K / X$ singular-equivalently, $M=L+K$ and $L \cap K \ll_{\delta} K$. The module $M$ is called $\delta$-supplemented if every submodule of $M$ has a $\delta$-supplement in $M$. On the other hand, the submodule $N$ is said to have ample $\delta$-supplements in $M$ if every submodule $L$ of $M$ with $M=N+L$ contains a $\delta$-supplement of $N$ in $M$. The module $M$ is called amply $\delta$-supplemented if every submodule of $M$ has ample $\delta$-supplements in $M$. Let $\mathbf{P}$ be the class of all singular simple modules. Let $M$ be any module. As in [7], let $\delta(M)=\operatorname{Rej}_{M}(\mathbf{P})=\bigcap\{N \leq M \mid M / N \in \mathbf{P}\}$. It is shown in [7, Lemma 1.5(1)] that $\delta(M)=\sum\left\{N \leq M \mid N \ll_{\delta} M\right\}$.

As in [3], a module $M$ is said to be $\delta$-local if $\delta(M) \ll_{\delta} M$ and $\delta(M)$ is a maximal submodule of $M$. For an $R$-module $M$, let $\operatorname{ann}(M)=\{r \in R \mid r M=0\}$ and for any $x \in M$, let $\operatorname{ann}(x)=\{r \in R \mid r x=0\}$. Let $L$ be a cyclic $\delta$-local module. Then $L \cong R / a$ with $a=\operatorname{ann}(L)$. Since $L$ is $\delta$-local, there exists a maximal ideal $m$ of $R$ such that $a \subseteq m$ and $\delta(R / a)=m / a$. In this case, we call the module $L m$ - $\delta$-local.

(C) 2012 Australian Mathematical Publishing Association Inc. 0004-9727/2012 \$16.00 


\section{Main results}

We begin with a lemma taken from [7, Lemmas 1.2, 1.3 and 1.5].

LeMMA 2.1. Let $M$ be a module.

(1) A submodule $N \leq M$ is $\delta$-small if and only if, for all submodules $X \leq M$, if $M=X+N$, then $M=X \oplus Y$ for a semisimple projective submodule $Y$ with $Y \subseteq N$.

(2) For submodules $N$ and $L$ of $M, N+L \ll_{\delta} M$ if and only if $N \ll_{\delta} M$ and $L \ll_{\delta} M$.

(3) If $K \ll_{\delta} M$ and $f: M \rightarrow N$ is a homomorphism, then $f(K) \ll_{\delta} N$. In particular, if $K \ll_{\delta} M \subseteq N$, then $K \ll_{\delta} N$.

(4) If $M=\bigoplus_{i \in I} M_{i}$, then $\delta(M)=\bigoplus_{i \in I} \delta\left(M_{i}\right)$.

(5) If $M$ is finitely generated, then $\delta(M) \ll_{\delta} M$.

It is well known that if $a$ is an ideal of $R$, then $a$ is essential in $R$ if and only if $R / a$ is a singular $R$-module (see, for example, [4, p. 32]).

Let $S$ be a simple $R$-module. Then $S$ is either singular or projective, but not both (see [4, Proposition 1.24]). Therefore $S$ is either $\delta$-local or projective.

A submodule $L$ of a module $M$ is called small in $M$ if $L+X \neq M$ for every proper submodule $X$ of $M$. Let $N$ be a submodule of a module $M$. A submodule $K$ of $M$ is called a supplement of $N$ in $M$ provided $M=N+K$ and $N \cap K$ is small in $K$. The module $M$ is called supplemented if every submodule of $M$ has a supplement in $M$. On the other hand, a submodule $N$ of a module $M$ has ample supplements in $M$ if every submodule $L$ such that $M=N+L$ contains a supplement of $N$ in $M$. The module $M$ is called amply supplemented if every submodule has ample supplements in $M$.

The following example shows that a $\delta$-supplemented module need not be amply $\delta$-supplemented.

ExAmple 2.2. Let $R$ be an incomplete discrete valuation ring with field of fractions $Q$. Then the $R$-module $Q \oplus Q$ is supplemented but not amply supplemented by [8, Theorem 2.2]. Let $m$ be the maximal ideal of $R$. Clearly $m$ is essential in $R$. Thus the simple $R$-module $R / m$ is singular. Hence every simple $R$-module is singular. So $R$ has no simple projective $R$-modules. Now let $N$ be a $\delta$-small submodule of an $R$-module $M$ and let $X$ be a submodule of $M$ with $N+X=M$. By Lemma 2.1(1), $M=Y \oplus X$ for a projective semisimple submodule $Y$ with $Y \subseteq N$. This clearly forces $Y=0$ and $X=M$. So $N$ is small in $M$. Consequently, the $R$-module $Q \oplus Q$ is $\delta$-supplemented but not amply $\delta$-supplemented.

LEMMA 2.3.

(1) If $a$ is a proper ideal of $R$, then the module $R / a$ is semisimple projective if and only if $a=\bigcap_{i=1}^{k} m_{i}$ is a finite intersection of nonessential maximal ideals $m_{i}(1 \leq i \leq k)$.

(2) For any module $M, M \ll_{\delta} M$ if and only if $M$ is semisimple projective.

Proof. (1) This follows from the Chinese remainder theorem.

(2) This follows from Lemma 2.1(1). 
Proposition 2.4. Let a be an ideal of $R$. The following conditions are equivalent:

(i) $\quad R / a$ is an $m$ - $\delta$-local module;

(ii) $m$ is essential in $R$ and is the only essential maximal ideal of $R$ which contains $a$.

Proof. (i) $\Rightarrow$ (ii) Suppose that $m$ is not essential in $R$. Then there exists a simple ideal $b$ of $R$ such that $m \oplus b=R$. Clearly, $(m / a) \oplus(b+a) / a=R / a$. Thus $(b+a) / a \nsubseteq$ $\delta(R / a)$ since $\delta(R / a)=m / a$. Hence the simple module $b \cong(b+a) / a$ is singular by Lemmas 2.3(2) and 2.1(3). That is, $R / m$ is a singular $R$-module. Therefore $m$ is essential in $R$, a contradiction. Now suppose that $R$ has an essential maximal ideal $m^{\prime} \neq m$ with $a \subseteq m^{\prime}$. Then $(R / a) /\left(m^{\prime} / a\right) \cong R / m^{\prime}$ is a singular $R$-module. So $\delta(R / a)=m / a \subseteq m^{\prime} / a$, a contradiction.

(ii) $\Rightarrow$ (i) Note first that $R / m \cong(R / a) /(m / a)$ is singular. Moreover, for any maximal ideal $m^{\prime}$ of $R$ with $m^{\prime} \neq m$ and $a \subseteq m^{\prime}, R / m^{\prime} \cong(R / a) /\left(m^{\prime} / a\right)$ is projective. Therefore $\delta(R / a)=m / a$. By Lemma 2.1(5), $\delta(R / a) \ll_{\delta} R / a$. So $R / a$ is $m-\delta$-local.

Let $m$ be a maximal ideal of $R$ and let $M$ be an $R$-module. Consider the subset $K_{\delta_{m}}(M) \subseteq M$ of elements $x \in M$ such that:

(i) $x=0$; or

(ii) $\quad m$ is essential in $R$ and is the only essential maximal ideal of $R$ which contains $\operatorname{ann}(x)$; or

(iii) $\operatorname{ann}(x)=\bigcap_{i=1}^{k} m_{i}$ such that each $m_{i}(1 \leq i \leq k)$ is a nonessential maximal ideal of $R$.

Proposition 2.5. Let $x$ be an element of a module $M$ and let $m$ be a maximal ideal of $R$. The following are equivalent:

(i) $x \in K_{\delta_{m}}(M)$;

(ii) $R / \operatorname{ann}(x)$ is $m$ - $\delta$-local or semisimple projective.

Proof. This is a consequence of Lemma 2.3 and Proposition 2.4.

Proposition 2.6. A factor module of an $m$ - $\delta$-local module is either $m$ - $\delta$-local or semisimple projective.

Proof. Let $a$ be an ideal of $R$ such that the $R$-module $R / a$ is $m-\delta$-local. Let $b$ be an ideal of $R$ with $a \subseteq b$. Note that $\delta(R / b) \ll_{\delta} R / b$ by Lemma 2.1(5). Consider the canonical epimorphism $\pi: R / a \rightarrow R / b$. We have $\pi(m / a)=(m+b) / b \ll_{\delta} R / b$ by Lemma 2.1(3). If $b \subseteq m$, then $m / b \ll_{\delta} R / b$. Therefore $m / b \subseteq \delta(R / b)$. This implies that $\delta(R / b)=R / b \ll_{\delta} R / b$ or $\delta(R / b)=m / b \ll_{\delta} R / b$. If $b \nsubseteq m$, then $R / b \ll_{\delta} R / b$. Therefore $R / b$ is semisimple projective or $m$ - $\delta$-local (see Lemma 2.3(2)).

Proposition 2.7. Let $M$ be an R-module and let $m$ be a maximal ideal of $R$. Then $K_{\delta_{m}}(M)$ is a submodule of $M$.

Proof. (1) Let us show that $K_{\delta_{m}}(M)$ is closed under multiplication by elements of $R$. Let $x \in K_{\delta_{m}}(M)$ and let $r \in R$. Let $a=\operatorname{ann}(x)$ and let $b=\operatorname{ann}(r x)$. Note that $a \subseteq b$. 
By Proposition $2.5, R / a$ is $m$ - $\delta$-local or semisimple projective. Note that $R / b \cong$ $(R / a) /(b / a)$. From Proposition 2.6 it follows that $R / b$ is $m$ - $\delta$-local or semisimple projective. So $r x \in K_{\delta_{m}}(M)$ by Proposition 2.5.

(2) Let us show that $K_{\delta_{m}}(M)$ is an additive subgroup of $M$. Let $x_{1}, x_{2} \in K_{\delta_{m}}(M)$, $a_{1}=\operatorname{ann}\left(x_{1}\right), a_{2}=\operatorname{ann}\left(x_{2}\right)$ and $a=\operatorname{ann}\left(x_{1}-x_{2}\right)$. Then $a_{1} \cap a_{2} \subseteq a$.

If $x_{1}=0$ or $x_{2}=0$, then of course $x_{1}-x_{2} \in K_{\delta_{m}}(M)$.

Suppose that $R / a_{1}$ is $m$ - $\delta$-local and $R / a_{2}$ is $m$ - $\delta$-local or semisimple projective. Since $m / a_{1}$ is essential in $R / a_{1}, m /\left(a_{1} \cap a_{2}\right)$ is essential in $R /\left(a_{1} \cap a_{2}\right)$ by [4, Proposition 1.1]. Moreover, if $a_{1} \cap a_{2} \subseteq m^{\prime}$ for some maximal ideal $m^{\prime} \neq m$, then $a_{1} \subseteq m^{\prime}$ or $a_{2} \subseteq m^{\prime}$. Therefore $m^{\prime}$ is not essential in $R$ (see Lemma 2.3 and Proposition 2.4). Thus $R /\left(a_{1} \cap a_{2}\right)$ is $m$ - $\delta$-local by Proposition 2.4. Since

$$
R / a \cong \frac{R /\left(a_{1} \cap a_{2}\right)}{a /\left(a_{1} \cap a_{2}\right)},
$$

$R / a$ is $m$ - $\delta$-local or semisimple projective by Proposition 2.6. So $x_{1}-x_{2} \in K_{\delta_{m}}(M)$ by Proposition 2.5.

Assume that both of $R / a_{1}$ and $R / a_{2}$ are semisimple projective. Note that $R x_{1}+$ $R x_{2}$ is semisimple projective. Since $R\left(x_{1}-x_{2}\right) \leq R x_{1}+R x_{2}, R / a \cong R\left(x_{1}-x_{2}\right)$ is semisimple projective. Thus $x_{1}-x_{2} \in K_{\delta_{m}}(M)$.

Recall that a submodule $N$ of a module $M$ is called cofinite if $M / N$ is finitely generated.

PRoposition 2.8. Let $M$ be a left module over any ring (not necessarily commutative). Suppose that every finitely generated submodule of $M$ is $\delta$-supplemented. Then every cofinite submodule of $M$ has ample $\delta$-supplements.

Proof. Let $N$ be a cofinite submodule of $M$ and let $L$ be a submodule of $M$ such that $M=N+L$. Then there exists a finitely generated submodule $F \leq L$ such that $M=N+F$. Consider the submodule $N \cap F \leq F$. By assumption, there exists a submodule $K \leq F \leq L$ such that $(N \cap F)+K=F$ and $N \cap K \ll_{\delta} K$. Since $N+K=M$, $K$ is a $\delta$-supplement of $N$ in $M$. This completes the proof.

THeOREM 2.9. Let $m$ be a maximal ideal of $R$ and $M$ be a module such that $K_{\delta_{m}}(M)=M$. Then every finitely generated submodule of $M$ is $\delta$-supplemented.

Proof. Let $x \in M$. Then $R x \cong R / \operatorname{ann}(x)$ is $m-\delta$-local or semisimple projective by Proposition 2.5. Therefore every finitely generated submodule of $M$ is a finite sum of $\delta$-local submodules and simple projective submodules. The result follows from [3, Proposition 3.5].

Corollary 2.10. Let $m$ be a maximal ideal of $R$. Let $M$ be a module such that $K_{\delta_{m}}(M)=M$. Then every cofinite submodule of $M$ has ample $\delta$-supplements.

Proof. This follows by Proposition 2.8 and Theorem 2.9. 
For any commutative ring $R$, let $\operatorname{Soc}_{P}(R)$ denote the sum of all simple projective ideals of $R$.

Lemma 2.11. Let a module $M=\bigoplus_{i \in I} M_{i}$ be a direct sum of submodules $M_{i}(i \in I)$. Assume that for all $i \neq j$ in $I, \operatorname{ann}\left(M_{i}\right)+\operatorname{ann}\left(M_{j}\right)$ is a direct summand of $R$ and $R /\left(\operatorname{ann}\left(M_{i}\right)+\operatorname{ann}\left(M_{j}\right)\right)$ is semisimple. Then, for every submodule $N$ of $M$,

$$
N \subseteq \bigoplus_{i \in I}\left(\left(N \cap M_{i}\right)+\operatorname{Soc}_{P}(R) M_{i}\right)
$$

Proof. Let $N$ be a submodule of $M$. Let $x \in N$. Then there exist a positive integer $n$, distinct elements $i_{j} \in I(1 \leq j \leq n)$ and elements $x_{j} \in M_{i_{j}}(1 \leq j \leq n)$ such that $x=x_{1}+\cdots+x_{n}$. If $n=1$, then $x=x_{1} \in N \cap M_{i_{1}}+\operatorname{Soc}_{P}(R) M_{i_{1}}$. Suppose that $n \geq 2$. By hypothesis, there exists a semisimple ideal $A_{1 n}$ of $R$ such that

$$
R=\left(\operatorname{ann}\left(M_{i_{1}}\right)+\operatorname{ann}\left(M_{i_{n}}\right)\right) \oplus A_{1 n} .
$$

So there exist elements $r, s$ and $t$ in $R$ such that $r x_{1}=0, s x_{n}=0, t \in A_{1 n}$ and $1_{R}=$ $r+s+t$. So

$$
\begin{aligned}
s x & =s x_{1}+s x_{2}+\cdots+s x_{n} \\
& =s x_{1}+s x_{2}+\cdots+s x_{n-1} \\
& =\left(1_{R}-r-t\right) x_{1}+s x_{2}+\cdots+s x_{n-1} \\
& =\left(1_{R}-t\right) x_{1}+s x_{2}+\cdots+s x_{n-1} .
\end{aligned}
$$

Note that $s x \in N,\left(1_{R}-t\right) x_{1} \in M_{i_{1}}$ and $s x_{j} \in M_{i_{j}}(2 \leq j \leq n-1)$. By induction on $n, \quad\left(1_{R}-t\right) x_{1} \in N \cap M_{i_{1}}+\operatorname{Soc}_{P}(R) M_{i_{1}}$. Thus $x_{1} \in N \cap M_{i_{1}}+\operatorname{Soc}_{P}(R) M_{i_{1}}+A_{1 n} M_{i_{1}}$. Clearly $A_{1 n} \subseteq \operatorname{Soc}_{P}(R)$. Hence $x_{1} \in N \cap M_{i_{1}}+\operatorname{Soc}_{P}(R) M_{i_{1}}$. In the same manner we can prove that $x_{j} \in N \cap M_{i_{j}}+\operatorname{Soc}_{P}(R) M_{i_{j}}(2 \leq j \leq n)$.

Lemma 2.12. Let $M$ be any module. Then $\operatorname{Soc}_{P}(R) M$ is a semisimple projective module. In particular, $\operatorname{Soc}_{P}(R) M \subseteq \operatorname{Soc}(M)$.

Proof. Let $S$ be a simple projective ideal of $R$. Then there exists a maximal ideal $m$ of $R$ such that $S \cong R / m$. Let $x \in M$ and let $\alpha \in S$. It is clear that $m(\alpha x)=0$. So $m \subseteq \operatorname{ann}(\alpha x)$. Thus $R(\alpha x)=0$ or $R(\alpha x) \cong R / m$ is simple projective. It follows that $S M$ is semisimple projective. Therefore $\operatorname{Soc}_{P}(R) M$ is semisimple projective.

Proposition 2.13. Let a module $M=M_{1} \oplus \cdots \oplus M_{n}$ be a finite direct sum of submodules $M_{i}(1 \leq i \leq n)$, for some positive integer $n \geq 2$. Assume that for all $1 \leq i<j \leq n, \operatorname{ann}\left(M_{i}\right)+\operatorname{ann}\left(M_{j}\right)$ is a direct summand of $R$ and $R /\left(\operatorname{ann}\left(M_{i}\right)+\operatorname{ann}\left(M_{j}\right)\right)$ is semisimple. If finitely generated submodules of $M_{i}$ are $\delta$-supplemented for all $1 \leq i \leq n$, then finitely generated submodules of $M$ are $\delta$-supplemented. 
Proof. Let $N$ be a finitely generated submodule of $M$. By Lemma 2.11,

$$
N \subseteq \bigoplus_{i=1}^{n}\left(\left(N \cap M_{i}\right)+\operatorname{Soc}_{P}(R) M_{i}\right)
$$

That is,

$$
N \subseteq\left(\bigoplus_{i=1}^{n}\left(N \cap M_{i}\right)\right)+\operatorname{Soc}_{P}(R) M
$$

Therefore

$$
N+\operatorname{Soc}_{P}(R) M=\left(\bigoplus_{i=1}^{n}\left(N \cap M_{i}\right)\right)+\operatorname{Soc}_{P}(R) M .
$$

Since $N$ is finitely generated, there exist finitely generated submodules $K_{i} \leq N \cap M_{i}$ $(1 \leq i \leq n)$ such that

$$
N+\operatorname{Soc}_{P}(R) M=\left(\bigoplus_{i=1}^{n} K_{i}\right)+\operatorname{Soc}_{P}(R) M .
$$

By hypothesis, the $K_{i}(1 \leq i \leq n)$ are $\delta$-supplemented. Since $\operatorname{Soc}_{P}(R) M$ is $\delta$ supplemented, $N+\operatorname{Soc}_{P}(R) M$ is $\delta$-supplemented by [6, Proposition 3.5]. As $\operatorname{Soc}_{P}(R) M$ is semisimple, $N$ is a direct summand of $N+\operatorname{Soc}_{P}(R) M$. So $N$ is $\delta$ supplemented by [6, Proposition 3.6].

Lemma 2.14. Let $a, b, c$ and $d$ be ideals of the ring $R$ such that $a \subseteq c$ and $b \subseteq d$. If $c / a \ll_{\delta} R / a$ and $d / b \ll_{\delta} R / b$, then $(c \cap d) /(a \cap b) \ll_{\delta} R /(a \cap b)$.

Proof. Let $u$ be an ideal of $R$ containing $a \cap b$ such that $(c \cap d) /(a \cap b)+u /(a \cap b)=$ $R /(a \cap b)$ and $R / u$ is singular. Then

$$
((c \cap d)+a) / a+(u+a) / a=R / a \text { and }((c \cap d)+b) / b+(u+b) / b=R / b .
$$

Hence $(c / a)+(u+a) / a=R / a$ and $(d / b)+(u+b) / b=R / b$. Moreover,

$$
\frac{R / a}{(u+a) / a} \cong \frac{R / u}{(u+a) / u} \text { and } \frac{R / b}{(u+b) / b} \cong \frac{R / u}{(u+b) / u} .
$$

Thus $(R / a) /((u+a) / a)$ and $(R / b) /((u+b) / b)$ are singular modules by [4, Proposition 1.22(b)]. By hypothesis, $R=u+a=u+b$. So $R=R R=(u+a)(u+b)=$ $u+a b$. But $a b \subseteq a \cap b \subseteq u$. Then $u=R$. This completes the proof.

Proposition 2.15. Let $m_{1}$ and $m_{2}$ be maximal ideals of $R$ with $m_{1} \neq m_{2}$. Let a module $M=M_{1} \oplus M_{2}$ such that $M_{1}$ is a finite direct sum of cyclic $m_{1}-\delta$-local submodules and $M_{2}$ is a finite direct sum of cyclic $m_{2}-\delta$-local submodules. Then $a=\operatorname{ann}\left(M_{1}\right)+$ $\operatorname{ann}\left(M_{2}\right)$ is a direct summand of $R$ and $R / a$ is semisimple. 
Proof. Assume that $M_{1}=\bigoplus_{i=1}^{k_{1}}\left(R / a_{1 i}\right)$ and $M_{2}=\bigoplus_{i=1}^{k_{2}}\left(R / a_{2 i}\right)$, where the $a_{1 i}(1 \leq$ $\left.i \leq k_{1}\right)$ and $a_{2 i}\left(1 \leq i \leq k_{2}\right)$ are ideals of $R$ such that $R / a_{1 i}\left(1 \leq i \leq k_{1}\right)$ are $m_{1}-\delta$-local modules and $R / a_{2 i}\left(1 \leq i \leq k_{2}\right)$ are $m_{2}$ - $\delta$-local modules. Then $\operatorname{ann}\left(M_{1}\right)=\bigcap_{i=1}^{k_{1}} a_{1 i}$ and $\operatorname{ann}\left(M_{2}\right)=\bigcap_{i=1}^{k_{2}} a_{2 i}$. Note that $m_{1} / a_{1 i} \ll_{\delta} R / a_{1 i}\left(1 \leq i \leq k_{1}\right)$ and $m_{2} / a_{2 i} \ll_{\delta} R / a_{2 i}$ $\left(1 \leq i \leq k_{2}\right)$. Therefore

$$
m_{1} /\left(\bigcap_{i=1}^{k_{1}} a_{1 i}\right) \ll_{\delta} R /\left(\bigcap_{i=1}^{k_{1}} a_{1 i}\right) \text { and } m_{2} /\left(\bigcap_{i=1}^{k_{2}} a_{2 i}\right) \ll_{\delta} R /\left(\bigcap_{i=1}^{k_{2}} a_{2 i}\right)
$$

by Lemma 2.14. That is, $m_{1} / \operatorname{ann}\left(M_{1}\right) \ll_{\delta} R / \operatorname{ann}\left(M_{1}\right)$ and $m_{2} / \operatorname{ann}\left(M_{2}\right) \ll_{\delta} R / \operatorname{ann}\left(M_{2}\right)$. By Lemma 2.1(3), $\left(m_{1}+a\right) / a \ll_{\delta} R / a$ and $\left(m_{2}+a\right) / a \ll_{\delta} R / a$. Thus

$$
\left(m_{1}+m_{2}+a\right) / a \ll_{\delta} R / a
$$

by Lemma 2.1(2). So $R / a \ll_{\delta} R / a$. By Lemma 2.3, $R / a$ is semisimple projective. Therefore $a$ is a direct summand of $R$ by [2, Proposition 17.2].

Proposition 2.16. Let $m_{1}$ be a maximal ideal of $R$ and let a module $M=M_{1} \oplus M_{2}$ be such that $M_{1}$ is a finite direct sum of cyclic $m_{1}-\delta$-local submodules and $M_{2}$ is a finite direct sum of simple projective submodules. Then $b=\operatorname{ann}\left(M_{1}\right)+\operatorname{ann}\left(M_{2}\right)$ is a direct summand of $R$ and $R / b$ is semisimple.

Proof. Assume that $M_{1}=\bigoplus_{i=1}^{k_{1}}\left(R / b_{1 i}\right)$ and $M_{2}=\bigoplus_{i=1}^{k_{2}}\left(R / m_{2 i}\right)$, where the $b_{1 i}(1 \leq$ $\left.i \leq k_{1}\right)$ are ideals of $R$ and $m_{2 i}\left(1 \leq i \leq k_{2}\right)$ are maximal ideals of $R$ such that $R / b_{1 i}$ $\left(1 \leq i \leq k_{1}\right)$ are cyclic $m_{1}-\delta$-local and $R / m_{2 i}\left(1 \leq i \leq k_{2}\right)$ are simple projective. Then $\operatorname{ann}\left(M_{1}\right)=\bigcap_{i=1}^{k_{1}} b_{1 i}$, ann $\left(M_{2}\right)=\bigcap_{i=1}^{k_{2}} m_{2 i}$ and $b=\left(\bigcap_{i=1}^{k_{1}} b_{1 i}\right)+\left(\bigcap_{i=1}^{k_{2}} m_{2 i}\right)$. Note that $m_{1} / b_{1 i} \ll_{\delta} R / b_{1 i}$ for all $1 \leq i \leq k_{1}$. It follows from Lemma 2.14 that $m_{1} /\left(\bigcap_{i=1}^{k_{1}} b_{1 i}\right) \ll_{\delta}$ $R /\left(\bigcap_{i=1}^{k_{1}} b_{1 i}\right)$. By Lemma 2.1(3), $\left(m_{1}+b\right) / b \ll_{\delta} R / b$. Suppose that $b \subseteq m_{1}$. Then $\bigcap_{i=1}^{k_{2}} m_{2 i} \subseteq m_{1}$. Hence $m_{2 j} \subseteq m_{1}$ for some $1 \leq j \leq k_{2}$. We thus get $m_{2 j}=m_{1}$. Therefore $R / m_{1}$ is projective. This contradicts the fact that $m_{1}$ is essential in $R$ (see Proposition 2.4 and [4, Proposition 1.24]). It follows that $b \nsubseteq m_{1}$ and hence $m_{1}+b=R$. Then $R / b \ll_{\delta} R / b$. By Lemma 2.3, $R / b$ is semisimple projective. Therefore $b$ is a direct summand of $R$ by [2, Proposition 17.2].

Proposition 2.17. Let a module $M=M_{1} \oplus \cdots \oplus M_{n}$ be a finite direct sum of submodules $M_{i}(1 \leq i \leq n)$, for some positive integer $n$ such that each $M_{i}$ is either cyclic $\delta$-local or simple projective. Then every finitely generated submodule of $M$ is $\delta$-supplemented.

Proof. By rearranging the submodules $M_{i}(1 \leq i \leq n)$, we can suppose that $M=$ $L_{1} \oplus \cdots \oplus L_{k}$ such that for every $1 \leq i \leq k-1, L_{i}$ is a finite direct sum of cyclic $m_{i^{-}}$ $\delta$-local submodules and $L_{k}$ is a finite direct sum of simple projective submodules, where $m_{1}, \ldots, m_{k-1}$ are distinct maximal ideals of $R$. Clearly, for every $1 \leq i \leq k-1$, $K_{\delta_{m_{i}}}\left(L_{i}\right)=L_{i}$ (see Propositions 2.5 and 2.7). By Theorem 2.9, finitely generated 
submodules of $L_{i}(1 \leq i \leq k)$ are $\delta$-supplemented. By Propositions 2.13, 2.15 and 2.16, finitely generated submodules of $M$ are $\delta$-supplemented.

Lemma 2.18. Let $M$ be a left module over any ring (not necessarily commutative). Assume that every finitely generated submodule of $M$ is $\delta$-supplemented. If $N$ is a homomorphic image of $M$, then every finitely generated submodule of $N$ is $\delta$-supplemented.

Proof. By assumption, there exists an epimorphism $f: M \rightarrow N$. Let $K$ be a finitely generated submodule of $N$. Then there exist a positive integer $n$ and elements $b_{i} \in N(1 \leq i \leq n)$ such that $K=R b_{1}+\cdots+R b_{n}$. Then there exist elements $a_{i} \in M$ $(1 \leq i \leq n)$ such that $f\left(a_{i}\right)=b_{i}(1 \leq i \leq n)$. Therefore $K=f\left(R a_{1}+\cdots+R a_{n}\right)$. Since $R a_{1}+\cdots+R a_{n}$ is $\delta$-supplemented, $K$ is $\delta$-supplemented by [6, Proposition 3.6].

Lemma 2.19. If $M$ is a $\delta$-local module, then $M=N \oplus L$ such that $N$ is a cyclic $\delta$-local submodule and $L$ is semisimple projective.

Proof. Let $M$ be a $\delta$-local module. Let $x \in M-\delta(M)$. As $\delta(M)$ is a maximal submodule of $M$, we have $\delta(M)+R x=M$. Since $\delta(M) \ll_{\delta} M$, there exists a semisimple projective submodule $L \leq \delta(M)$ such that $L \oplus R x=M$ (see Lemma 2.1(1)). By Lemma 2.1(4), $\delta(M)=\delta(L) \oplus \delta(R x)$. From Lemma 2.3(2) it follows that $\delta(L)=L$. Thus $\delta(M)=L \oplus \delta(R x)$. Therefore $\delta(R x)$ is a maximal submodule of $R x$. Moreover, according to Lemma 2.1(5), $\delta(R x) \ll_{\delta} R x$. Consequently, $N=R x$ is a cyclic $\delta$-local module.

Theorem 2.20. Let $M$ be a module such that $M$ is a sum of $\delta$-local submodules and a semisimple projective submodule. Then every finitely generated submodule of $M$ is $\delta$-supplemented.

Proof. By Lemma 2.19, there is no loss of generality in assuming that $M=\sum_{i \in I} M_{i}$ such that $M_{i}$ is either cyclic $\delta$-local or a simple projective submodule of $M$. Let $K$ be a finitely generated submodule of $M$. There exists a finite subset $J \subseteq I$ such that $K \leq \sum_{i \in J} M_{i}$. By Proposition 2.17, every finitely generated submodule of the module $\bigoplus_{i \in J} M_{i}$ is $\delta$-supplemented. Since $\sum_{i \in J} M_{i}$ is a homomorphic image of $\bigoplus_{i \in J} M_{i}, K$ is $\delta$-supplemented by Lemma 2.18. This completes the proof.

Corollary 2.21. Let $M$ be a module such that $M$ is a sum of $\delta$-local submodules and a semisimple projective submodule. Then every cofinite submodule of $M$ has ample $\delta$-supplements.

Proof. This follows by Proposition 2.8 and Theorem 2.20.

Lemma 2.22. Let $N$ be a maximal submodule of a module $M$. If $K$ is a $\delta$-supplement of $N$ in $M$, then $K$ is either $\delta$-local or semisimple projective. 
Proof. By assumption, $N+K=M$ and $N \cap K \ll_{\delta} K$. Thus $N \cap K \subseteq \delta(K)$. Since $M / N \cong K /(N \cap K), N \cap K$ is a maximal submodule of $K$. Hence $\delta(K)=N \cap K$ or $\delta(K)=K$. If $\delta(K)=N \cap K$, then $K$ is $\delta$-local. Now suppose $\delta(K)=K$. Then for every $x \in K-(N \cap K), R x+(N \cap K)=K$. Moreover, since $R x \subseteq \delta(K)$ and $\delta(K)=\sum\{L \leq K \mid$ $\left.L \ll_{\delta} K\right\}, R x \ll_{\delta} K$ by Lemma 2.1(2). Again by Lemma 2.1(2), $R x+(N \cap K)=$ $K \ll_{\delta} K$. Therefore $K$ is semisimple projective by Lemma 2.3.

A module $M$ is called coatomic if every proper submodule of $M$ is contained in a maximal submodule of $M$.

Corollary 2.23. Let $M$ be a coatomic module. Suppose that every cofinite submodule of $M$ has a $\delta$-supplement in $M$. Then:

(1) every finitely generated submodule of $M$ is $\delta$-supplemented;

(2) every cofinite submodule of $M$ has ample $\delta$-supplements.

Proof. (1) By [1, Theorem 2.9] and Lemma 2.22, $M$ is a sum of $\delta$-local submodules and a semisimple projective submodule. The result follows from Theorem 2.20.

(2) Use (1) and Proposition 2.8.

COROllary 2.24. Any finitely generated $\delta$-supplemented module is amply $\delta$-supplemented.

Proof. This follows by Corollary 2.23.

We conclude this paper by noting that there are some types of rings, not necessarily commutative, over which finitely generated $\delta$-supplemented modules are amply $\delta$-supplemented.

EXAMPLE 2.25.

(1) It is easily seen that if a ring $R$ is semisimple or right artinian, then all finitely generated modules are amply $\delta$-supplemented.

(2) In [7], Zhou called a ring $R \delta$-semiperfect if every left ideal $I$ of $R$ can be written as $I=R e \oplus S$, where $e^{2}=e \in R$ and $S \subseteq \delta\left({ }_{R} R\right)$. From [5, Theorem 3.3] and Proposition 2.8 it follows that finitely generated modules over $\delta$-semiperfect rings are amply $\delta$-supplemented.

\section{References}

[1] K. Al-Takhman, 'Cofinitely $\delta$-supplmented and cofinitely $\delta$-semiperfect modules', Internat. J. Algebra 1(12) (2007), 601-613.

[2] F. W. Anderson and K. R. Fuller, Rings and Categories of Modules (Springer, New York, 1974).

[3] E. Büyükaşik and C. Lomp, 'When $\delta$-semiperfect rings are semiperfect', Turkish J. Math. 34 (2010), 317-324.

[4] K. R. Goodearl, Ring Theory: Nonsingular Rings and Modules (Marcel Dekker, New York, 1976).

[5] M. T. Koşan, ' $\delta$-lifting and $\delta$-supplemented modules', Algebra Colloq. 14(1) (2007), 53-60.

[6] Y. Wang, ' $\delta$-small submodules and $\delta$-supplemented modules', Int. J. Math. Math. Sci. (2007), Article ID 58132, 8p. 
[7] Y. Zhou, 'Generalizations of perfect, semiperfect, and semiregular rings', Algebra Colloq. 7(3) (2000), 305-318.

[8] H. Zöschinger, 'Komplemente als direkte Summanden', Arch. Math. 25 (1974), 241-253.

RACHID TRIBAK, Centre Pédagogique Régional (CPR) - Tanger,

Avenue My Abdelaziz, Souani, BP : 3117, Tangier, Morocco

e-mail: tribak12@yahoo.com 